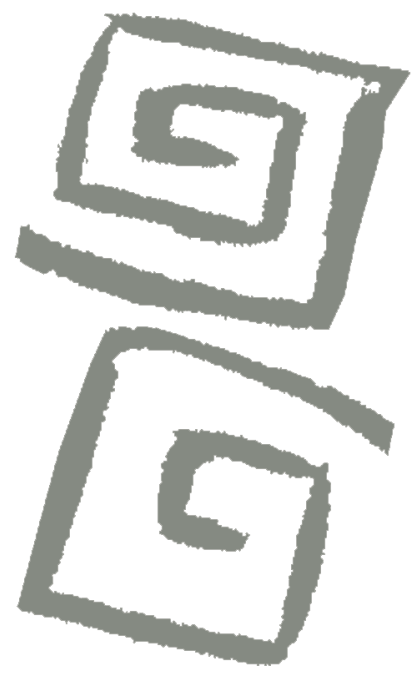

\title{
Políticas de drogas en el contexto brasileño: un análisis interseccional de "Cracolandia" en San Pablo, Brasil
}

\author{
Drug policies in the Brazilian context: an intersectional \\ analysis of "Cracolândia" in São Paulo, Brazil
}

Ana Lucia Marinho Marques ${ }^{1}$, Marcia Thereza Couto ${ }^{2}$

${ }^{1}$ Autora de correspondencia. Doctora en Salud Colectiva. Terapeuta ocupacional, Faculdade de Medicina Universidade de São Paulo, São Paulo, Brasil. $\bowtie$ iD

${ }^{2}$ Doctora en Sociología. Posdoctora en Salud Colectiva. Profesora, Departamento de Medicina Preventiva y Programa de Pós-Graduación em Saúde Coletiva, Faculdade de Medicina, Universidade de São Paulo, São Paulo, Brasil. $\triangle$ iD
RESUMEN A partir de un contexto específico como es la zona de "Cracolandia", en San Pablo, Brasil, y el programa denominado "De Braços Abertos", este artículo analiza las complejas relaciones que se establecen entre el consumo de drogas y la formulación de políticas públicas dirigidas a la atención de usuarios. En términos metodológicos, el trabajo se basa en una investigación cualitativa, en la que se realizaron entrevistas semiestructuradas a trece informantes claves. El material empírico se analizó desde el análisis temático de contenido y desde la perspectiva de la interseccionalidad, a partir de las contribuciones teóricas de la categoría de "diferencia". Los resultados señalan "diferencias" marcadas por género, raza y posición que los sujetos ocupan en el tejido social, reforzando los estigmas y sus diferentes impactos en las relaciones sociales. Este documento contribuye al debate sobre la necesidad de formular diferentes enfoques para las necesidades y demandas específicas de las poblaciones o categorías de clasificación social con respecto a las políticas públicas sobre drogas.

PALABRAS ClAVES Política Pública; Políticas Públicas de Salud; Reducción del Daño; Cocaína Crack; Interseccionalidad; Brasil.

ABSTRACT From the analysis of a specific conjuncture, "Cracolândia" in São Paulo, Brazil, and the "De Braços Abertos" program, this article discusses the complex relationships between drug use and the formulation of public policies directed to the care of users. In methodological terms, this work is based on the qualitative research by using semistructured interviews with thirteen key informants. The empirical material was analyzed from the content thematic analysis and the intersectionality perspective, especially from the theoretical contributions of the "difference" category. The results point out that "differences" are marked by gender, race and social position in the society, reinforcing stigmas and their different impacts on social relations. This paper contributes to the debate on the need to formulate different approaches to the specific needs and demands of populations or social classification categories regarding drug public policy.

KEY WORDS Public Policy; Public Health Policies; Harm Reduction; Crack Cocaine; Intersectionality; Brazil. 


\section{INTRODUCCIÓN}

Desde la segunda mitad del siglo XIX, en el debate sobre las drogas como un "problema social" para el Estado y la sociedad, y la consiguiente formulación de políticas de drogas como campo de acción gubernamental, han confluido una serie de factores religiosos, políticos, económicos y morales, entre otros, que no pueden ser considerados de forma aislada, dado que han conformado una compleja trama de fuerzas y producciones discursivas ${ }^{(1,2,3,4)}$.

Como políticas de drogas se entiende al conjunto de leyes y programas que tienen por objetivo influir en la decisión de los individuos con relación al consumo de sustancias psicoactivas e intervenir sobre los impactos y consecuencias de ese consumo relacionados con la seguridad, la salud y el bienestar social de los individuos y las Comunidades ${ }^{(5)}$. Babor et al. ${ }^{(5)}$ consideran que las políticas de drogas actuales abarcan una amplia gama de medidas administrativas que pueden ser agrupadas en tres categorías: 1) los programas destinados a la prevención; 2) los programas de salud y asistencia social dirigidos al cuidado de los usuarios; y 3) el conjunto de leyes que prohíben o reglamentan el uso, la tenencia, la distribución y la producción de determinadas sustancias que establecen sanciones a la violación de esas prohibiciones.

La discusión actual sobre las políticas de drogas en América Latina ha llevado a los gobiernos a repensar los abordajes tradicionales en este campo; particularmente, aquellos que defienden solo la reducción de la oferta con el objetivo de adecuar las intervenciones a las dinámicas y culturas locales de sus países y a las problemáticas específicas de la región ${ }^{(6,7)}$. Como presupuesto básico para este debate está la premisa de que la denominada "guerra contra las drogas", pautada por la prohibición, control y represión al uso, tráfico y producción de determinadas sustancias psicoactivas no solo fracasó, sino que produjo, como principal consecuencia, una profunda y extensa desigualdad expresada en el alto costo social y económico resultante de la violencia asociada al combate del tráfico de dro$\operatorname{gas}^{(7,8,9)}$. Diversas investigaciones muestran que las estrategias centradas en la disminución de la oferta han aumentado los índices de violencia con el consiguiente incremento de las tasas de homicidio, la corrupción de instituciones y agentes estatales, el aumento de la población carcelaria y la superpoblación en las prisiones, así como violaciones a los derechos humanos ${ }^{(10,11,12)}$. Comprender el impacto del prohibicionismo y de sus efectos en América Latina es fundamental para que esta región pueda construir sus propias estrategias y políticas basadas en sus realidades locales y en sus propios saberes, con la intención de superar el abordaje coercitivo por un abordaje que valore la construcción de espacios de cohesión social ${ }^{(7,13,14)}$. En ese sentido, se destaca la estrategia de reducción de daños que, entre sus premisas fundamentales, coloca la perspectiva de la salud como punto central de las problemáticas vinculadas a las drogas. En América Latina, el horizonte conceptual de la reducción de daños se amplió, constituyéndose como un campo ético-político que considera fundamental la intervención en los contextos donde viven las personas, así como la necesidad de reducir la violencia y las desigualdades desencadenadas por la guerra contra las drogas, anclando la discusión del cuidado en el fortalecimiento y la garantía de los derechos sociales y humanos ${ }^{(6,9,15,16)}$.

En Brasil, a contramano de las directrices recomendadas internacionalmente y basadas en evidencias científicas, en junio de 2019 se sancionó la Ley Federal 13.840 sobre el Sistema Nacional de Políticas Públicas sobre Drogas, que modifica la legislación anterior, y que ha sido considerada un retroceso, especialmente en lo que atañe a las acciones de cuidado en salud dirigidas a las personas que hacen un uso abusivo del alcohol y de otras drogas. Entre los puntos críticos, destacamos el rechazo a reconocer la reducción de daños como abordaje y directriz para el cuidado, centrándose en el presupuesto de la abstinencia como única meta a ser alcanzada a través del "tratamiento" de las personas, y 
la centralidad de las internaciones como estrategia, con la aprobación de decretos que aumentan la transferencia de fondos hacia hospitales psiquiátricos y la instauración de mecanismos de financiamiento específicos para las comunidades terapéuticas.

En ese sentido, cabe destacar que bajo un determinado discurso médico y la necesidad de "tratamiento" se reiteran estrategias de segregación y aislamiento que favorecen el aumento del estigma y la marginalización de los usuarios de drogas ${ }^{(13,17)}$. Además, las comunidades terapéuticas han sido bastante cuestionadas como estrategia de "tratamiento" y "rehabilitación" en América Latina, y han sido denunciadas por la sistemática violación de los derechos humanos, por medio de prácticas descriptas como: privación de la libertad, castigos, puniciones, humillación pública, contención, prácticas religiosas obligatorias y trabajo forzado, entre otras ${ }^{(18,19,20)}$.

En ese contexto sociopolítico y considerando la relevancia social otorgada al uso del crack en Brasil, y la importancia de visibilizar experiencias y proyectos desarrollados en el país y que busquen abordar esa cuestión de forma abarcadora, equilibrada y basada en los derechos, este artículo tiene por objetivo discutir el contexto de proposición de políticas dirigidas a personas usuarias de crack a partir del análisis de "Cracolandia", espacio-territorio usualmente mencionado para describir las "escenas de uso" o el "flujo" de personas que compran y consumen crack en las calles de la región de Luz, en el centro de San Pablo. Específicamente, analiza el período de desarrollo del programa denominado De Braços Abertos (DBA), que estuvo en vigencia entre 2014 y 2017.

El análisis del caso de "Cracolandia" y de las políticas formuladas para responder a las cuestiones que se presentan en ese contexto resultan significativas para la comprensión de las complejas relaciones que se establecen entre consumo de drogas, las profundas desigualdades que caracterizan a la sociedad brasileña y la formulación de políticas públicas dirigidas al cuidado de personas que usan drogas. En esa dirección, y buscando un abordaje que permita aprehender la complejidad de las relaciones entre sujetos, agentes, estructuras, marcadores sociales e instituciones, el referencial teórico metodológico elegido fue el de la interseccionalidad.

Las nociones de "interseccionalidades" o "categorías articuladas", expresiones usadas a veces como sinónimos, son comprendidas en este trabajo como las relaciones entre "múltiples dimensiones y modalidades de relaciones sociales y formaciones subjetivas" ${ }^{\prime \prime 21)}$ y los resultados de las interacciones, en términos de poder, entre categorías y marcadores de la diferencia en las prácticas sociales, identidades y subjetividades, acuerdos institucionales e ideologías culturales ${ }^{(22)}$.

El término "interseccionalidad" fue utilizado por primera vez en 1989, por la abogada y teórica feminista estadunidense Kimberlé Crenshaw ${ }^{(23)}$, con el objetivo de "capturar las consecuencias estructurales y dinámicas de la interacción entre dos o más ejes de subordinación", buscando tratar también la forma en que las "acciones y políticas específicas generan opresiones que fluyen a lo largo de tales ejes, constituyendo aspectos dinámicos o activos del desempoderamiento"(24).

Considerada como una de las cuatro perspectivas representativas de la tercera ola del feminismo (junto con los abordajes posestructuralistas y posmodernos, de la teoría feminista poscolonial y de la agenda de la "generación joven") (25), o incluso hasta como "la más importante contribución del campo de estudios feministas"(21), la interseccionalidad fue ganando fuerza en las décadas siguientes, y se observa una creciente popularidad, expansión y articulación con nuevas fronteras del conocimiento ${ }^{(26,27)}$.

Al situar este estudio en el campo de la salud colectiva, es importante resaltar la creciente relevancia de la interseccionalidad como referencial teórico-metodológico en investigaciones en ese campo. Estas buscan comprender la articulación de los marcadores sociales en cuestiones complejas que involucran tanto el proceso salud-enfermedad-cuidado como también el análisis de programas e instituciones de la salud, debido a su potencial para anclar discusiones preocupadas en evidenciar posibilidades de formulación de 
políticas y organización de programas y servicios de salud comprometidos con la justicia social y la garantía de derechos ${ }^{(28)}$.

Piscitelli ${ }^{(29)}$ presenta una buena síntesis de lo que significa una propuesta de trabajo en esta perspectiva: ofrecer herramientas analíticas para aprehender la articulación de múltiples diferencias y desigualdades. Es importante destacar que no se trata de la diferencia sexual, ni de la relación entre género y raza o género y sexualidad, sino de la diferencia, en un sentido amplio. En ese sentido, la interseccionalidad debe ser comprendida como una pregunta abierta, que solo puede ser respondida en contextos específicos y a partir de investigaciones concretas ${ }^{(30)}$. El análisis que se pretende desarrollar a partir de esta perspectiva puede desligarse de la obligatoriedad de partir de un marcador social específico, y adoptar una postura abierta y atenta para captar las articulaciones entre aquellos que se muestran relevantes o, como plantea Henning(31), "las diferencias que hacen la diferencia" en determinados contextos históricos, socioculturales y políticos.

A fin de profundizar esta comprensión, destacamos la contribución de Avtar Brah ${ }^{(32)}$, elegida como referencial analítico de ese trabajo. El concepto de diferencia, para esta autora, se refiere a la variedad de maneras en que se constituyen los discursos específicos de la diferencia, y cómo son discutidos, reproducidos y resignificados. Algunas construcciones de la diferencia postulan fronteras fijas e inmutables entre determinados grupos sociales mientras otras construcciones pueden presentar la diferencia como relacional, contingente y variable. Considerando que, a priori, la diferencia no siempre será un marcador de jerarquía ni de opresión, cabe a quien investiga cuestionarse permanentemente si la diferencia se remitirá a la desigualdad, la opresión, la explotación, el igualitarismo, la diversidad o las formas democráticas de agencia política. Entonces, las cuestiones a ser analizadas no se refieren a la "diferencia" en sí, sino a quién define la diferencia, cómo se representan las categorías y los grupos dentro de los discursos de la "diferencia" y si se postula una diferencia lateral o jerárquica ${ }^{(29,32)}$.

\section{RECORRIDO METODOLÓGICO}

Los datos empíricos y el análisis que sustentan las discusiones en este artículo forman parte de una investigación mayor, cuyo objetivo fue describir y analizar los procesos de formulación y gestión de políticas públicas orientadas a la atención y el cuidado de personas que usan drogas en el municipio de San Pablo, Brasil(33).

La investigación adoptó un abordaje cualitativo, que se caracteriza por su carácter comprensivo, contextualizado, interpretativo y dialéctico ${ }^{(34)}$. La producción de los datos empíricos se basó en la técnica de entrevista en profundidad, debido a su potencialidad de abordar, a partir de una guía predefinida flexible, los significados de las intencionalidades de los actores sociales como también los aspectos estructurales involucrados en los procesos sociales estudiados ${ }^{(35)}$.

El diseño de la investigación contempló delinear una muestra por conveniencia con informantes claves. El conjunto de participantes estuvo compuesto por personas involucradas de forma significativa con la formulación de políticas, programas y prácticas enfocadas en la construcción de respuestas para los problemas relacionados con el uso de alcohol y otras drogas en el municipio de San Pablo, Brasil.

Se elaboró una lista inicial de posibles participantes con el objetivo de alcanzar un encuadre amplio, con múltiples voces (gestores, trabajadores del campo de la salud y de la asistencia social, investigadores, participantes de asociaciones de usuarios de drogas y representantes de movimientos y organizaciones sociales) que pudieran ofrecer narrativas construidas a partir de lugares y puntos de vista diferentes. Luego de cada entrevista, se revisó la lista inicial de informantes, y para complementar la información o comprender algún aspecto inicialmente no previsto, se utilizó la estrategia de reclutamiento en cadena o "bola de nieve"(36), buscando conformar una muestra no probabilística, en la que los participantes del estudio indicaran a otros participantes. 
Desde septiembre de 2017 a junio de 2019 se realizaron trece entrevistas (ocho hombres y cinco mujeres). Si bien se privilegió la realización de forma presencial, cuando no fue posible o por pedido del participante se realizó vía Skype, con un tiempo medio de duración de 90 minutos. Todas las entrevistas fueron grabadas y transcriptas por completo y, a continuación, se verificó la precisión de las transcripciones. De acuerdo con las normas del Consejo Nacional de Salud del Brasil, relativas a las investigaciones con seres humanos, el proyecto fue presentado ante el Comité de Ética en Investigación de la Facultad de Medicina de la Universidad de San Pablo (CEP-FMUSP) y aprobado bajo el expediente No. 2.056.573. Todos los sujetos entrevistados aceptaron y confirmaron su participación por medio de la firma de un documento que explicaba los términos de la investigación y dieron su consentimiento de forma libre e informada.

El proceso de análisis del material empírico se realizó secuencialmente, aplicando el análisis de contenido temático ${ }^{(37,38)}$. Luego de reiteradas lecturas y de la delimitación de los temas prioritarios para el análisis (temas previstos en la guía de entrevistas y emergentes), el proceso de interpretación de los datos se basó en la perspectiva interseccional y en el referencial analítico propuesto por Brah ${ }^{(32,39)}$, que propone trabajar la "diferencia" como categoría analítica y sugiere cuatro modos de conceptualización, que comprenden la diferencia como: experiencia, relación social, subjetividad e identidad. A partir de esa conceptualización, la autora coloca en un primer plano de análisis la articulación de los diferentes elementos a ser considerados, en los que la dicotomía micro-macro no es entendida como niveles opuestos, sino como inherentes a los procesos de articulación por los cuales los discursos y las prácticas inscriben las relaciones sociales, las posiciones del sujeto y las subjetividades. En otras palabras, no se trata de privilegiar "macro" y "micro" como niveles de análisis, sino de identificar y analizar las relaciones entre afectos, subjetividades, contextos, relaciones sociales y políticas $^{(32,39)}$.

\section{RESULTADOS}

\section{"Cracolandia" paulista y políticas públicas municipales dirigidas al cuidado de personas que usan drogas}

Desde comienzos de la década de 1990, hay registros de la presencia de consumidores de crack en la región central de la ciudad de San Pablo, especialmente en las calles del céntrico barrio denominado Luz. Con el pasar de los años, junto al aumento de la concentración de usuarios en esa región y la construcción social y mediática del crack como peligro público y amenaza social, este espacio-territorio pasó a ser denominado "Cracolandia". Esta área se tornó conocida por la ocupación y por el flujo de personas que consumían crack y en situación de calle, llamadas "nóias" (acortamiento de paranoia) de manera peyorativa y "crackeros". Todos ellos asociados a la "degradación" del espacio urbano y al aumento de la criminalidad y la violencia en la región ${ }^{(40,41,42,43,44)}$.

Considerado el mayor y más famoso espacio abierto de uso de crack en Brasil, "Cracolandia" ha sido el centro de intervenciones estatales desde su surgimiento. A lo largo de las décadas de 1990 y 2000, se formularon diversas iniciativas basadas, principalmente, en la represión a los usuarios a través de operaciones amenazadoras por parte de los organismos de seguridad pública municipal y del estado, así como la revitalización del espacio urbano, tanto con la realización de reformas estructurales de edificios y espacios culturales, como con expropiaciones y demoliciones de establecimientos como conventillos, pensiones y hoteles ${ }^{(40,41,42,43,44)}$.

A lo largo de la década de 2000, se invirtió en la ampliación de la red de servicios de salud en esa región, y se implementaron equipos de salud especializados para el abordaje y el seguimiento de personas en situación de calle, servicios de atención psicosocial comunitaria y territorial con funcionamiento ininterrumpido, servicios de urgencia, ambulatorios especializados, unidades transitorias de acogida y camas en hospitales generales y especializados. Se invirtió, también, en la 
implementación de equipos y se amplió la red de servicios de asistencia social ${ }^{(45)}$.

Sin embargo, ni la represión ni la oferta de servicios de salud y socioasistenciales fueron suficientes para enfrentar la cuestión. En ese contexto, a partir de iniciativas de aproximación, vínculo, escucha y negociación entre agentes de servicios socioasistenciales, gestores y la población a ser atendida, se implementó el programa De Braços Abertos ${ }^{(44,46,47)}$. Con el objetivo de promover la rehabilitación psicosocial de personas en situación de vulnerabilidad social y el uso abusivo de sustancias psicoactivas, a través de la promoción de derechos y de acciones asistenciales, de salud y de prevención del uso abusivo de drogas, el programa De Braços Abertos se caracterizó por la implementación de acciones intersectoriales y articuladas entre las políticas municipales de salud, derechos humanos, asistencia social, trabajo, seguridad urbana, educación, vivienda, deporte, cultura y medio ambiente, entre otras ${ }^{(48)}$.

Desde el punto de vista programático, el programa De Braços Abertos es consistente con el abordaje conocido internacionalmente como "housing first" (vivienda primero), que enfatiza la provisión de vivienda permanente y estable como estrategia primaria para las personas en situación de calle, incluso aquellas con cuestiones de salud mental y uso de drogas. Diversos estudios señalan la asociación entre la adopción de ese abordaje con la disminución en el consumo de alcohol y otras drogas, de la violencia y de la supuesta percepción de desorden urbano debido al exceso de conflictos en las calles. Otro concepto fundamental y asociado es la "baja exigencia", que implica no condicionar la oferta de la vivienda a la abstinencia o a la obligatoriedad de estar en tratamientos como forma de preparación o "calificación", enfatizando el acceso a la vivienda como un derecho fundamental ${ }^{(44,46,47,49,50)}$.

El abordaje del "housing first" surgió en la década de 1990, en EEUU, y su metodología y principios han sido implementados en experiencias desarrolladas y relatadas en ciudades de Europa y Canadá, principalmente. En América Latina, aunque aún su adopción no sea significativa, diversos proyectos en curso comparten algunas características del housing first ${ }^{(51)}$.

La estructuración del programa se basó, en primer lugar, en la transferencia de las personas que dormían en casillas improvisadas y que ocupaban las veredas de las calles en el centro de la ciudad hacia los hoteles de la región, alquilados por la Prefectura para albergar a los beneficiarios del programa. Además de vivienda asegurada y alimentación en un restaurante popular del barrio, se crearon fuentes de trabajo con pagos semanales, y la garantía del acceso al cuidado y a la atención integral en salud ${ }^{(44,46,47)}$.

En 2015, se desarrolló una evaluación preliminar del programa, en la cual se buscó conocer el perfil de los beneficiarios y sus percepciones sobre las acciones desarrolladas. Entre los datos relevados, destacamos que la mayoría de las personas atendidas tenían más de 30 años $(77 \%)$, con hijos $(73 \%)$, habían pasado por el sistema penitenciario $(67 \%)$ y se encontraban en zonas de vulnerabilidad o desafiliación social| ${ }^{(52)}$, con bajos índices de escolaridad, inserción precaria en el mercado de trabajo y redes sociales fragilizadas. También había un número mayor de hombres: el $58 \%$ declaró ser del sexo masculino, el 37\% del sexo femenino y el $5 \%$ transexuales. En cuanto a la autodeclaración racial/étnica, el $68 \%$ de los beneficiarios se autodeclaró pardo/mestizo y negro y, el $23 \%$, blanco ${ }^{(44)}$. Tales datos merecen atención en el proceso de formulación de políticas, dado que son similares a los datos nacionales sobre personas en situación de calle que usan crack, en los que se observa una superposición y sobrerrepresentación de marcadores de diferencia, desigualdad y exclusión social, en comparación con los datos de la población brasileña en general ${ }^{(53)}$.

\section{Las diferencias que conforman la formulación de políticas de drogas}

Las drogas son productos cuyas cualidades farmacológicas son virtualmente insignificantes fuera de sus contextos político-económicos y 
socioculturales. Las representaciones sociales sobre drogas se desarrollan en determinados contextos sociales e históricos, forjando ideologías propias de cada droga. Cada sustancia, en cada contexto, carga sentidos específicos, que atraviesan tanto el fenómeno del consumo como del control y las prohibiciones ${ }^{(54,55)}$. En el caso del crack, en Brasil, las representaciones construidas y reforzadas mediáticamente, están asociadas a la idea de "una droga-basura (lo que sobra de la cocaína refinada), utilizada por personas descalificadas en términos sociales, que se aglomeran en centros urbanos" ${ }^{\prime(56)}$.

Al ser rotuladas como "crackeras", las identidades de las personas que usan crack y que viven o circulan por las calles de la región central paulistana quedan anuladas y reducidas a la sustancia que consumen. La asociación a esa "droga-basura", articulada a la marginalidad y a la delincuencia, construye discursivamente una diferencia que coloca en una posición de inferioridad y descalifica a esa población como "descartables urbanos" $^{\prime(57)}$, y pone a los individuos en una situación jurídica excepcional, en la que al mismo tiempo son "culpables" y "enfermos"(58).

En ese contexto, esa es la diferencia que se visibiliza como un problema a ser enfrentado, justificando las intervenciones gubernamentales, predominantemente fundamentadas tanto por un discurso jurídico-moral que criminaliza los comportamientos y justifica la represión, como por el discurso médico-psiquiátrico que patologiza las experiencias y reitera la necesidad de represión y segregación, pero en "ambientes especializados" como hospitales psiquiátricos y comunidades terapéuticas, que tienen como meta la "cura" y la "abstinencia".

En un primer plano, el uso abusivo de crack y otras drogas es el marcador evidente que fundamenta el proceso de formulación de políticas públicas. En el proceso de presentar las iniciativas que dieron lugar a la implementación del programa De Braços Abertos, lo que diferencia la visibilización de ese marcador con relación a las políticas formuladas anteriormente es la adopción de la perspectiva del uso de crack como un problema social complejo, y su articulación con la situación de segregación social de las personas que viven en "Cracolandia".

En ese campo, es importante preguntarse: ¿política para quién? ¿De qué usuario en situación abusiva estoy hablando? Y la construcción política que hicimos en el "De Braços Abertos" estuvo dirigida a personas de altísima vulnerabilidad social en situación de calle y que hacen uso abusivo de droga. (Interlocutora 11)

El tema de las drogas como política pública tiene el desafío de lidiar con poblaciones muy vulnerables, marginalizadas, excluidas y que Jessé Souza denomina "ralé" [escoria]. La discusión de la "ralé" es más determinante en la cuestión de las drogas que la droga en sí. (Interlocutor 4)

La adopción de esta perspectiva, planteada por la mayoría de nuestros interlocutores, llevó a considerar que no es el uso de crack en sí lo que debe ser visibilizado como problema prioritario a ser enfrentado, sino el estatus de ciudadanía de las personas y sus necesidades de reproducción social. Por lo tanto, la atención en ese campo exige políticas sociales integradas, que no estén basadas solo en la oferta de servicios sanitarios y socioasistenciales. Esa es la premisa que lleva al diseño inicial del programa De Braços Abertos, según los interlocutores que estuvieron involucrados de forma más significativa en ese proceso.

Entonces profundizamos en que no era una cuestión solo de salud o solo de seguridad o solo de asistencia social, sino que era una cuestión de mucha complejidad, y no había ninguna solución simple para ese problema complejo. (Interlocutora 13)

...quien acompañó el desarrollo de las políticas ahí en la región de Luz, se dio cuenta de que una política de salud sola no iba a alcanzar porque había una dimensión de la miseria que no iba ser resuelta. [...] Hay que resolver las 
cuestiones de vivienda y de generación de ingresos. (Interlocutor 9)

Es importante señalar que la inclusión de la ciudadanía y de la defensa de los derechos sociales y humanos como foco y eje del trabajo va al encuentro de las directrices éticas de la reducción de daños, cuyas primeras experiencias en América Latina datan de fines de la década de 1990, no solo como estrategia de trabajo en salud dirigida a usuarios de drogas sino, fundamentalmente, centrada en la modificación de prácticas de riesgos relacionadas con la transmisión del VIH. Con el pasar de los años, si bien las estrategias se fueron ampliando y se aplicaron en diversos programas desarrollados en la región, tienen en común la preocupación por cuestiones como violencia, pobreza y desigualdades sociales, y buscan construir prácticas que respondan a problemas sociales concretos, que intervengan en las condiciones de vida de los sujetos y en las estructuras que los (re)producen. En ese sentido, pasa a ser fundamental la incorporación de las nociones de territorio, comunidad y trabajo en red en la formulación de prácticas y políticas sociales dirigidas al cuidado de esa población ${ }^{(15,59,60)}$.

Durante las entrevistas, la forma en que los entrevistados percibían las diferencias en el proceso de formulación de políticas sobre drogas movilizó diversas narrativas que englobaban distintas temáticas y posibilidades de conceptualización de la diferencia. La referencia al racismo, por ejemplo, apareció no solo como una relación social sistemática y que posiciona a los sujetos en relaciones de poder desiguales, sino también como una experiencia subjetiva y desencadenante de sufrimiento psíquico, muchas veces ignorado por los profesionales en su abordaje clínico hacia los usuarios.

En el proceso de análisis del material empírico, partimos de una diferencia construida como forma de categorizar y caracterizar un grupo social ("usuarios de crack en situaciones de vulnerabilidad y segregación social") y con una mirada atenta a su heterogeneidad interna y a las relaciones de poder que atraviesan a ese grupo. Considerando los principales énfasis planteados por los entrevistados, en este artículo, destacamos dos categorías que, de acuerdo con Brah ${ }^{(32)}$, pueden ser consideradas como dos formas de conceptualización de la diferencia: diferencia como identidad y diferencia como relación social.

\section{Diferencia como identidad}

Para $\mathrm{Brah}^{(32)}$, las identidades se inscriben a través de experiencias culturalmente construidas en relaciones sociales y están marcadas "por la multiplicidad de posiciones de sujeto que constituyen al sujeto". Así, las identidades personales se articulan con las experiencias colectivas de un grupo, pero la singularidad de las experiencias cotidianas y relaciones sociales vividas producen trayectorias que no reflejan la experiencia del grupo, del mismo modo que las identidades colectivas no son reductibles a la suma de las experiencias individuales.

En este sentido, y considerando la noción de diferencia como identidad propuesta por Brah, lo que resultó más evidente y fue más explorado por los participantes con relación al grupo de beneficiarios del programa De Braços Abertos fue la diferencia entre "hombres" y "mujeres", desde la identificación de esta diferencia como marcador identitario (género) hasta la descripción de las prácticas de cuidado diferenciadas (o prácticas generificadas), desarrolladas para ellos. En otros términos, la visibilización de esa diferencia revela su interpretación de la identidad de género, traducida en expectativas y percepciones -de acuerdo con el contexto sociocultural en que están insertos- vinculadas a los modos de organización y pertenencia al género masculino y femenino ${ }^{(61)}$.

Al identificar y poner en primer plano tanto esa diferencia de performatividad de género, como también las características hegemónicamente asociadas a la feminidad o a la masculinidad, la "maternidad", en sus diferentes aspectos, emergió como uno de los temas principales que marcó tanto la identificación de demandas como la formulación de 
prácticas diferenciadas para las beneficiarias del programa. La asociación entre uso de drogas e incapacidad para el cuidado autónomo en sus modos de llevar la vida repercute en la noción, bastante difundida socialmente, de que las mujeres usuarias de drogas no tendrían la capacidad de cuidar a sus hijos, lo que genera mucha discusión en el campo de la formulación de políticas sobre la intervención estatal de abrigo y tutela de niñas y niños. En ese sentido, en las entrevistas se destacan tanto la preocupación por este aspecto como la necesidad de que sea más visibilizado y discutido para producir nuevas prácticas.

En esta política, especialmente, creo que un marcador importante es género, porque no hay un recorte de género hasta hoy y para evitar que la única política sea la de sacarle los bebés a las madres. Entonces se necesita una política que ayude a esas mujeres a fortalecerse $y$ organizarse, tal vez ir a un lugar en el que puedan vivir con sus compañeros o solas, que puedan quedarse un tiempo después con sus hijos, etc. (Interlocutora 1)

Con relación al desarrollo de prácticas vinculadas al cuidado en salud de las mujeres que forman parte del programa, las entrevistas plantearon un desafío inicial: establecer estrategias diferenciadas para su seguimiento. Las estrategias convencionales y recomendadas para el cuidado realizado por los equipos de salud no se mostraban eficaces para una gran parte de esa población. Por lo tanto, fue necesario invertir en estrategias que viabilizaran la construcción del cuidado a partir del trabajo realizado en la calle por los equipos de salud y de asistencia social

En ese campo, identificamos que se le dio especial importancia a la salud reproductiva de las mujeres y se invirtió en la formulación de abordajes que posibilitaran la reflexión sobre gestación y formas de protección, desde acceso a la información hasta la oferta de métodos anticonceptivos considerados más adecuados para las mujeres que trajeron esa demanda.
Conseguimos hacer el prenatal, conseguiríamos hacer prevención con estas mujeres, y utilizar métodos anticonceptivos, esos implantes de larga duración [...] porque ella no va a tomar todos los días el comprimido. (Interlocutora 13)

Para el momento específico del embarazo, y considerando la importancia del seguimiento prenatal, los equipos de salud establecieron diversos abordajes. A partir de las necesidades y demandas de cuidados que involucran a la mujer embarazada y al bebé, y de su evaluación como un momento de mayor vulnerabilidad, se señaló la preocupación por establecer un servicio de vivienda específico para esa población y, en el proceso de implementación de un hotel localizado en una región más alejada del centro del municipio de San Pablo, se priorizó la derivación de las mujeres embarazadas.

...y creamos el hotel "Freguesia do Ó", a partir de una demanda por parte de los beneficiarios de salir del territorio, pero teniendo como prioridad la derivación de las mujeres embarazadas... allá estábamos con unas 30 mujeres embarazadas en aquel momento. (Interlocutora 2)

En general, aunque parte de los interlocutores hayan usado la expresión "género", la referencia a esa categoría quedó marcada, muchas veces, como sinónimo de "mujer", y asociada solo a las necesidades y demandas específicas de las mujeres, mientras que los hombres se mantuvieron invisibilizados en sus necesidades específicas. Las percepciones sobre las diferencias entre "hombres" y "mujeres" aquí destacadas ponen en evidencia la dimensión relacional entre estas categorías y la necesaria comprensión de género como una categoría relacional, transversal, instauradora y reproductora de asimetrías de poder ${ }^{(62,63,64)}$.

La percepción sobre esta diferencia aparece, también, bastante asociada a comprender a las mujeres en situación de calle como "más vulnerables" que los hombres. Esa percepción de mayor vulnerabilidad, o desventaja, justificaría la formulación y organización de 
prácticas dirigidas a una atención diferenciada de las mujeres y a la disminución de barreras y desigualdades para el acceso al cuidado.

Dentro de esa altísima vulnerabilidad de Luz [referencia al barrio], nosotros teníamos grupos más vulnerables. ¿Qué grupo era más vulnerable? El de las mujeres. (Interlocutora 11)

...yo no pienso en un servicio solo para mujeres que usan drogas. Pienso mucho más en dispositivos que estén dentro de un servicio, por ejemplo, dentro de un CAPS de alcohol y drogas [servicio comunitario de salud mental, alcohol y drogas], que reciban las demandas que son de las mujeres que usan drogas. Entonces, por ejemplo, un grupo o algún otro tipo de estrategia, que esté dirigida a las mujeres que usan drogas y que tienen hijos. Ellas necesitan ir, por ejemplo, a una consulta. Ellas no tienen con quien dejar a su hijo. Si dentro del propio servicio no hay un espacio que garantice que ella pueda ir y dejar el hijo ahí con alguien mientras va a la consulta, ella puede dejar de ir, simplemente, porque el servicio no garantiza una demanda que ella tiene. (Interlocutor 7)

Las categorías "mujeres" y "hombres", en general, referidas a ese contexto específico, aparecían en los relatos de los participantes como categorías "atomizadas" y desvinculadas de otras categorías de diferenciación como edad, raza, origen, sexualidad, escolaridad. Pero hubo una excepción: la diferencia ubicada en la intersección entre identidad de género y sexo gonadal, identificada, en la práctica, en las experiencias de las mujeres transexuales. Una interlocutora, que destacó especialmente esa diferencia, estaba preocupada por establecer estrategias de organización de los servicios específicamente dirigidos a esa población como, por ejemplo, la definición de habitaciones y baños diferenciados en hoteles y centros de acogida con el propósito no solo de afirmar la diversidad sino también de disminuir desventajas en el acceso a los derechos y servicios.

La cuestión de las trans es muy importante porque, primero, no tienen un lugar adecuado en la sociedad [...] esa población en sí ya sufre una desventaja y una dificultad de acceso como un todo en las políticas públicas, entonces es obvio que hay que tener un recorte [...] Por ejemplo, había una habitación de uno de los hoteles que era específicamente de las trans... si bien no se estableció un flujo claro y determinado para eso, fue sucediendo y nosotros entendíamos que tenía esa importancia. (Interlocutora 2)

Considerando las diferencias aquí enumeradas, cabe reflexionar sobre cómo se visibilizaron en este contexto, y sus aportes al debate sobre ciudadanía y derechos. El movimiento por la formulación de políticas y estrategias focalizadas en algunas identidades socialmente construidas puede no solo favorecer la afirmación de la diversidad y de las singularidades político-culturales, sino señalar las oportunidades para aquellos que viven discriminaciones, desigualdades y opresiones en sus experiencias y relaciones sociales $^{(65,66,67)}$.

La discusión crítica acerca de la ciudadanía lleva también a cuestionar los derechos que, al universalizarse, terminan invisibilizando y excluyendo. Esto lleva a cuestionar a ese sujeto único que se afirma y se constituye sobre la negación de tantos otros, y a poner en discusión las políticas de identidad, más como una estrategia y un posicionamiento, que como un fin en sí mismo ${ }^{(68,69)}$.

\section{Diferencia como relación social}

El concepto de "diferencia como relación social", según Brah ${ }^{(32)}$ se refiere "a la manera en que la diferencia se constituye y organiza en relaciones sistemáticas a través de discursos económicos, culturales y políticos y prácticas institucionales". 
El concepto de diferencia, en el sentido propuesto, destaca la articulación de regímenes de poder, históricamente variable, dentro de los cuales se estructuran determinados modos de diferenciación. Sin privilegiar o dar centralidad a lo "estructural", como un eje que determina las experiencias, considera que los efectos de las relaciones sociales pueden ser observados en todos los espacios de formación social: en las políticas, en las instituciones, en las subjetividades, en los afectos y en los escenarios donde se desarrolla la vida cotidiana concretamente ${ }^{(32,39)}$.

Dentro de la categorización de la diferencia, entendida como relación social, el primer punto a destacar a partir de los relatos de los interlocutores es la necesidad de deconstruir la noción, bastante difundida socialmente, de que el uso de crack habría sido el principal factor de desestabilización y desorganización de la vida de las personas y, por lo tanto, responsable de su recorrido de pérdidas materiales y afectivas que culminaron con la ruptura de vínculos sociofamiliares y la "elección" de una vida de privación y en situación de calle. Por el contrario, otros señalan la importancia de conocer las trayectorias y las experiencias de los sujetos que, en ese momento particular de la vida, circulan en el "flujo", usan crack y viven en situación de calle. Además de la importancia de la comprensión de las singularidades de las experiencias individuales, plantean la necesidad de reconocer los contextos y las relaciones que caracterizan esas trayectorias y la situación de vida de ese grupo de personas. En ese contexto, el énfasis en los discursos se situó en la importancia de comprender cómo determinados regímenes de poder se articulan en la producción de diferencias que, por medio de relaciones sistemáticas, reproducen vulnerabilidad, opresiones y desigualdades en la vida de las personas.

Esas personas que están en Cracolandia no se hicieron vulnerables a causa del uso de la droga. Ellas vienen de una altísima vulnerabilidad social, la gran mayoría, claro que hay excepciones, pero vienen de una gran vulnerabilidad social y están insertas en este contexto por esa vulnerabilidad. (Interlocutora 11)

Hoy, el crack, en Brasil, es un problema social. El impacto que tiene en la sociedad y, por lo tanto, la razón para priorizar las políticas es la combinación con la miseria y la desigualdad, con relaciones bastante complejas, que sirven a un discurso de criminalización indirecto y/o de culpabilización de sujetos por su pobreza, diciendo que elegir el crack lo hizo pobre, es lo que lo mantiene en la pobreza. (Interlocutor 9)

Al comprender la dimensión de la "miseria" y de la "pobreza" como eje y foco de intervenciones y políticas dirigidas a los usuarios abusivos de crack en situación de calle, la referencia al marcador social de "clase" aparece interseccionado al marcador "raza". En diversos relatos en los que se mencionaron esos marcadores, se visibilizó la importancia de desnaturalizar las desigualdades que conforman la sociedad brasileña y de analizar el racismo como estructural, estructurante e indisociable de los mecanismos de clasificación/desclasificación social.

La cuestión racial en el país es muy importante, porque tiene que ver con la cuestión económica, pero también con ciertos comportamientos. El problema de Brasil es el cruce de una práctica esclavista con el capitalismo. [...] Nuestra cultura esclavista está enraizada en nuestra cuestión de la desigualdad. O, al contrario, yo diría: nuestra desigualdad está enraizada en una estructura esclavista. (Interlocutor 4)

...la discusión de la política de drogas necesita tener un recorte de raza: a las personas que más detienen son negras, o las que más mueren son negras, y eso se vincula con las relaciones sociales $y$ 
económicas establecidas en Brasil que es un país fundado en un pacto racista. (Interlocutora 1)

En el proceso de reflexión sobre la identificación de diferencias que estructuran relaciones de desigualdad en ese contexto, junto con la dimensión de la miseria, la violencia fue planteada como una cuestión a ser visibilizada. Es fundamental que la discusión sobre la violencia se haga de forma compleja, problematizando los efectos de las políticas prohibicionistas, que contribuyeron a que América Latina se transformara en la región más violenta del planeta, medida por los niveles de homicidios y ejecuciones extrajudiciales, detenciones arbitrarias y por la falta de acceso a servicios básicos de salud. La región también registra la tasa de homicidio juvenil más alta del mundo, superando a países y regiones en guerra ${ }^{(6,70,71)}$.

Castro-Gómez ${ }^{(72)}$ advierte sobre los procesos de formación de los Estados-nación latinoamericanos, permeados por la perpetuación y acentuación de la violencia con el objetivo del control y de la gestión poblacional. Salvando las singularidades históricas de cada país, la región aún carga las marcas de la explotación colonial y las características de la violencia, usurpación, represión y exterminio de aquel período. Pero esas opresiones no ocurren en un plano abstracto, sino que se plasman en los cuerpos subalternizados ${ }^{(73)}$.

La violencia atraviesa los cuerpos y experiencias de los usuarios de crack, y se expresa en los abordajes policiales, en las relaciones con los agentes de tránsito, en los altos índices de homicidios que se configuran como su principal causa de muerte ${ }^{(74)}$. Los relatos que abordan específicamente la cuestión de la violencia en el territorio de "Cracolandia" expresan la percepción de que esta no incide de la misma forma sobre todas las personas que circulan por allí. Una vez más prevalece la preocupación por las mujeres, a las que consideran como sujetas a posiciones de mayor opresión en las relaciones que se establecen en ese contexto y más expuestas a situaciones de violencia física, verbal y sexual.
Y la cuestión de la violencia de género es muy grande, incluso en "De Braços Abertos" también sucedía; intentábamos aplacar, pero aun así sucedía mucho. Una vez, un hombre y una mujer se estaban peleando en la escena de uso... Y ahí fui a hablar con el Guardia Civil Metropolitano, para pedir ayuda, y él dijo: "Em briga de marido e mulher, ninguém mete a colher" [refrán popular "en pelea de marido y mujer nadie mete la cuchara"]. Eso está tan enraizado que muchas veces se produce un femicidio porque las personas no pueden entrometerse en una "pelea de marido y mujer". (Interlocutora 2)

Con relación a las mujeres transexuales, resalta la preocupación por la exposición a situaciones de violencia como algo muy presente en su cotidiano, asociadas al prejuicio y discriminación que ya sufren socialmente, en gran medida, por construir y performatizar su identidad de género asociada a lo femenino, así como contradecir el comportamiento socialmente esperado para su sexo gonadal.

Nosotros hicimos un trabajo con el centro de diversidad sexual aquí del centro, en todos los centros de acogida, en todos los núcleos de servicio de la población adulta de calle. De repente, creo que unos quince días después que hicimos ese trabajo con todos los servicios, recibimos una notificación de travestis que estaban siendo discriminadas por profesionales de un Centro de Referencia en Asistencia Social... ¡ ¡ómo nos íbamos a imaginar que nuestros profesionales iban a burlarse de travestis en situación de calle? (Interlocutor 3)

...por ejemplo, centro de acogida para trans... tienen una habitación allá en el centro de acogida Zachi Nachi y ellas sufren violencia ahí adentro también. (Interlocutora 2)

La distinción entre la violencia a la cual son sometidos los cuerpos de las mujeres, cis y transexuales, en ese contexto, parece 
fundamental desde una perspectiva que considere raza, clase, género y sexualidad como sistemas imbricados y mutuamente constitutivos. Plantear esa reflexión no significa, de ninguna manera, identificar cuál sería el grupo "más oprimido" sino señalar la necesidad de enfrentar esas dimensiones en conjunto ${ }^{(69,75)}$.

La identificación de esa diferencia y el análisis de cómo se presenta, nos lleva también a problematizar el estigma y el poder discrecional de los agentes institucionales que controlan el acceso a determinados recursos y servicios previstos en el contexto de una determinada política pública, que terminan orientando sus prácticas sobre la base de prejuicios sobre determinadas características de la población, que dificultan el acceso a aquellos que más necesitan del apoyo y la intervención del Estado para garantizar sus derechos y el ejercicio de la ciudadanía, creando desigualdades entre grupos e individuos $^{(76,77)}$.

\section{CONSIDERACIONES FINALES}

"Cracolandia" no es un espacio físico. Son personas. Personas singulares, hombres y mujeres, con sus historias y experiencias, que viven o solo transitan por aquella región, que consumen también otras drogas, que tienen afectos, relaciones, deseos y necesidades concretas. El análisis de las diferencias, en ese contexto específico, posibilita tanto el ejercicio de una mirada atenta a las singularidades como a la identificación de los sistemas y regímenes de poder que caracterizan las trayectorias de exclusión constitutivas de ese grupo.

Si la formulación de políticas y programas de acogida y cuidado dirigidos a personas usuarias de crack y otras drogas en situación de calle se planteara como una necesidad, el análisis de ese contexto específico, con tantas particularidades involucradas, permitiría afirmar la exigencia de formular políticas que aborden de forma integrada las relaciones entre consumo de drogas y segregación social, que intervengan en las condiciones de vida y la existencia de esa población. En ese sentido, el programa De Braços Abertos fue considerado un programa innovador $y$ con posibilidades de replicar sus conceptos y abordajes, respetando las singularidades de los contextos locales y regionales ${ }^{(46,47)}$. Es importante resaltar que esa exigencia se condice con la reducción de daños como directriz política, que considera la defensa del fin de la guerra a las drogas como punto de partida en el campo de las políticas de cuidado ${ }^{(9,59)}$. La reducción de daños, en esa dirección, se plantea como una perspectiva emancipadora, asentada en un proyecto de transformación social. Y como tal, es fundamental que esté siempre acompañada de manera atenta, crítica y cuestionadora ${ }^{(78)}$.

Considerando los límites de las políticas y prácticas universalistas, reflexionar sobre la diferencia en la formulación de las políticas dirigidas al cuidado de personas que usan drogas desde una perspectiva interseccional posibilita comprender tanto las relaciones entre los marcadores sociales y cómo estos se intersecan de modo dinámico y complejo con el uso de drogas ${ }^{(58)}$, como también dar respuesta a determinados grupos que reivindican atención diferenciada a sus demandas identitarias de acceso a derechos y garantía de ciudadanía ${ }^{(65)}$. En esta línea es importante tener cuidado de que al enfrentar una opresión identificada no se fortalezca otra. El desafío, entonces es formular estrategias para enfrentar las desigualdades de forma no compartimentada o jerarquizada, sino a partir de comprender cómo se interconectan y articulan, en un determinado contexto específico $^{(32)}$.

Las críticas al modelo bélico adoptado de forma hegemónica en América Latina como matriz de las políticas públicas sobre drogas se han constituido de forma amplia y articulada entre los movimientos sociales y políticos contemporáneos que luchan por la soberanía política, la independencia económica y la justicia social de la región ${ }^{(7)}$. En ese sentido, destacamos la oportunidad del diálogo con la producción de investigadores latinoamericanos que utilizan de forma crítica y contextualizada el abordaje de la interseccionalidad, destacando que el análisis 
de las desigualdades sociales debe considerar la heterarquía de los múltiples regímenes de poder que sitúan a las sociedades y los Estados locales en una situación de subordinación, en el contexto de las complejas relaciones de poder que conforman el sistema global capitalista ${ }^{(69,75)}$.

Por último, consideramos importante señalar la preocupación de que el reconocimiento de las diferencias y las singularidades da lugar a un discurso acentuado en la ética neoliberal, que busca eximir al Estado y responsabilizar a los sujetos por sus "competencias" individuales para el ejercicio de la ciudadanía y de la inclusión social ${ }^{(79)}$. En el contexto social y político actual, de fragilización de la democracia y de dudas en cuanto al papel del Estado, son muchos los desafíos para consolidar las políticas de drogas en Brasil y en toda América Latina, que opten por modelos no bélicos y se comprometan con la afirmación de una ética ciudadana que garantice derechos.

\section{REFERENCIAS BIBLIOGRÁFICAS}

1. Fiore M. A medicalização da questão do uso de drogas no Brasil: reflexos acerca de debates institucionais e jurídicos. En: Venâncio RP, Carneiro H, (orgs.). Álcool e drogas na história do Brasil. São Paulo: Alameda; 2005. p 257-290.

2. Rodrigues T. Narcotráfico: um esboço histórico: Álcool e drogas na história do Brasil. São Paulo: Alameda; 2005.

3. Carneiro H, Del Priore M. Pequena enciclopédia da história das drogas e bebidas: histórias e curiosidades sobre as mais variadas drogas e bebidas. São Paulo: Elsevier; 2005

4. Berridge V. Dependência: história dos conceitos e teorias. En: Edward G, Lader M, (eds.). A natureza da dependência de drogas. Porto Alegre: Artes Médicas; 1994.

5. Babor T, Caulkins J, Edwards G, Fischer B, Foxcroft D Humphreys K, Strang J. La política de drogas y el bien público. Washington DC: Organización Panamericana de la Salud; 2013.

6. Carvalho IS. O despertar da América Latina : uma revisão do novo debate sobre política de drogas. Rio Janeiro: Instituto Igarapé; 2014.

7. Ralón G. Consecuencias de la guerra contra las drogas. En: Touzé G, Goltzman P (comps.). América Latina debate sobre drogas: I y II Conferencias Latinoamericanas sobre Políticas de Drogas. Buenos Aires: Intercambios Asociación Civil; Facultad de Ciencias Sociales, Universidad de Buenos Aires; 2011.

8. Fraga PCP. A geopolítica das drogas na América Latina. Em Pauta: Teoria Social e Realidade Contemporânea. 2007;(19):67-88.
9. Menéndez EL. Sustancias consideradas adictivas: Prohibición, reducción de daños y reducción de riesgos. Salud Colectiva. 2012;8(1):9-24.

10. Dammert L, Zúñiga L. La cárcel: problemas y desafíos para las Américas. Santiago de Chile: Facultad Latinoamericana de Ciencias Sociales-Chile; 2008.

11. Institute Transnational on Latin America Washington Office. Sistemas sobrecargados: Leyes de drogas y cárceles en América Latina. Washington: Washington Office on Latin America; 2010.

12. Werb D, Rowell G, Guyatt G, Kerr T, Montaner J, Wood E. Effect of drug law enforcement on drug market violence: A systematic review. International Journal of Drug Policy. 2011;22(2):87-94.

13. United Nations Office on Drugs and Crime, Escritório das Nações Unidas sobre drogas e crime. Da coerção à coesão: Tratamento da dependência de drogas por meio de cuidados em saúde e não da punição. Documento para discussão com base em uma oficina técnica. Viena: United Nations Office on Drugs and Crime; 2009.

14. United Nations Office on Drugs and Crime. People at the centre: UNODC support for UNGASS 2016 on the world drug problem. Vienna: United Nations Office on Drugs and Crime; 2018.

15. Galante A, Rossi D, Goltzman P, Pawlowicz MP. Programas de reducción de daños en el escenario actual: Un cambio de perspectiva. Revista Escenarios. 2009;(14):113-121.

16. Petuco DR. Redução de danos: das técnicas à ética do cuidado. En: Ramminger T, Silva M, (orgs.). Mais substâncias para o trabalho em saúde com usuários de drogas. Porto Alegre: Rede Unida; 2014. p. 133-148. 
17. Ministério da Saúde, Coordenação Nacional de DST/ AIDS. A Política do Ministério da Saúde para a atenção integral a usuários de álcool e outras drogas. Brasil: Ministério da Saúde, Coordenação Nacional de DST/AIDS; 2003.

18. Tomasini-Joshi D. Ni Socorro, Ni Salud: Abusos en vez de rehabilitación para usuarios de drogas en América Latina y el Caribe. New York: Open Society Foundations; 2016.

19. Conselho Federal de Psicologia, Mecanismo Nacional de Prevenção e Combate à Tortura, Procuradoria Federal Dos Direitos do Cidadão, Ministério Público Federal. Relatório da Inspeção Nacional em Comunidades Terapêuticas. Brasil: Conselho Federal de Psicologia; 2018.

20. United Nations International Children's Emergency Fund, Oficina del Alto Comisionado de las Naciones Unidas para los Derechos Humanos. Compilación de observaciones finales del Comité de los Derechos del Niño sobre es de América Latina y el Caribe (19932006). Santiago de Chile: UNICEF, Oficina del Alto Comisionado para los Derechos Humanos; 2006.

21. McCall L. The complexity of Intersectionality. Signs: journal of Women in Culture and Society. 2005;30(3): 1771:1800. doi: 10.1086/426800.

22. Davis K. Intersectionality as buzzword: A sociology of science perspective on what makes a feminist theory successful. Feminist Theory. 2008;9(1):67-85. doi: $10.1177 / 1464700108086364$.

23. Crenshaw K. Mapping the Margins: Intersectionality, Identity Politics, and Violence against Women of Color. Stanford Law Review. 1991;43(6):1241-1299. doi: $10.2307 / 1229039$

24. Crenshaw K. Documento para o encontro de especialistas em aspectos da discriminação racial relativos ao gênero. Revista Estudos Feministas. 2002;10(1):171-188. doi: 10.1590/S0104-026X2002000100011.

25. Mann SA, Huffman DJ. The decentering of second wave feminism and the rise of the third wave. Science \& Society. 2005;69(1):56-91. doi: 10.1521/siso.69.1.56.56799.

26. Brah A, Phoenix A. Ain't I a woman? Revisiting intersectionality. Journal of International Women's Studies. 2004;5(3):75-86

27. Bilge S. De I'analogie à I'articulation : ThéOriser la différenciation sociale et l'inégalité complexe. L'Homme \& la Société. 2010;176-177(2):43-64. doi: 10.3917/ Ihs.176.0043.

28. Couto MT, De Oliveira E, Separavich MAA, Luiz ODC. La perspectiva feminista de la interseccionalidad en el campo de la salud pública: revisión narrativa de las producciones teórico-metodológicas. Salud Colectiva. 2019;15:e1994. doi: 10.18294/sc.2019.1994.

29. Piscitelli A. Interseccionalidades, categorias de articulação e experiências de migrantes brasileiras. Sociedade e Cultura. 2008:11(2):263-274.
30. Vigoya MV. La interseccionalidad: una aproximación situada a la dominación. Debate Feminista. 2016;52:117. doi: 10.1016/j.df.2016.09.005.

31. Henning CE. Interseccionalidade e pensamento feminista: as contribuições históricas e os debates contemporâneos acerca do entrelaçamento de marcadores sociais da diferença. Mediações. Revista de Ciências Sociais. 2015;20(2):97-128. doi: 10.5433/2176-6665.2015 v20n2p97.

32. Brah A. Diferença, diversidade, diferenciação. Cadernos Pagu. 2006;26:329-376. doi: 10.1590/S010483332006000100014

33. Marques ALM. Políticas públicas de cuidado dirigidas a pessoas que usam drogas no município de São Paulo/SP: uma análise desde a perspectiva da interseccionalidade [Tesis de Doutorado]. São Paulo: Faculdade de Medicina; 2019. doi:10.11606/T.5.2020. tde-11032020-110156.

34. Minayo MCDS. Origen de los argumentos científicos que fundamentan la investigación cualitativa. Salud Colectiva. 2017;13(4):561-575. doi: 10.18294/sc.2017.942.

35. Becker HS. Métodos de pesquisa em ciências sociais. São Paulo: HUCITEC; 1993.

36. Noy C. Sampling knowledge: The hermeneutics of snowball sampling in qualitative research. International Journal of Social Research Methodology. 2008;11(4):327344. doi: 10.1080/13645570701401305.

37. Bardin L. Análise de Conteúdo. São Paulo: Edições 70; 2011

38. Minayo MCDS, Deslandes SF, Neto OC, Gomes R. Pesquisa Social: Teoria, método e criatividade. Rio de Janeiro: Editora Vozes; 2011.

39. Brah A. Cartografías de la diáspora: Identidades en cuestión. Revista de Estudios Sociales. 2014;(49):223225. doi: 10.7440/res49.2014.19.

40. Frugoli Junior H. Da cracolândia aos nóias: percursos etnográficos no bairro da Luz. Ponto Urbe. 2010;4(6). doi: 10.4000/pontourbe.1870.

41. Gomes BR, Adorno CF. Tornar-se "noia": trajetória e sofrimento social nos "usos de crack" no centro de São Paulo. Etnografica. 2011;15(3):569-586. doi: 10.4000/ etnografica. 1076.

42. Nasser MMS. Entre a ameaça e a proteção: categorias, práticas e efeitos de uma política de inclusão na Cracolândia de São Paulo. Horizontes Antropológicos. 2018;24(50):243-270. doi: 10.1590/s0104-71832018000 100009 .

43. Rui T. Depois da "Operação Sufoco": sobre espetáculo policial, cobertura midiática e direitos na "cracolândia" paulistana. Contemporânea. 2013;3(2):287-310.

44. Rui T, Fiore M, Tófoli LF. Pesquisa preliminar de avaliação do Programa "De Braços Abertos". En: Plataforma Brasilera de Política Drogas [Internet]. Brasil: 2016. 
[citado 17 jun 2019]. Disponible en: https://tinyurl.com/ y792vgt2.

45. Prefeitura de São Paulo. Instrumento normativo para monitoramento técnico, administrativo e financeiro dos serviços de saúde mental no Município de São Paulo. São Paulo: Secretaria Municipal de Saúde; 2012.

46. Evans S. Crack: Reduzir Danos: Lições Brasileiras de Saúde, Segurança, e Cidadania. New York: Open Society Foundations; 2017.

47. Carvalho IS, Pellegrino AP. Políticas de Drogas no Brasil: a mudança já começou. Rio de Janeiro: Instituto Igarapé; 2015

48. Brasil, Prefeitura de São Paulo. Decreto № 55.067 de 28 de abril de 2014. Diário Oficial Cidade de São Paulo [Internet]. 2014 [citado 10 may 2019]. Disponible en: https://tinyurl.com/y93webo3.

49. Marlatt GA, Larimer ME, Witkiewitz K. Harm reduction: Pragmatic strategies for managing high-risk behaviors. London: Guilford Press; 2011.

50. Tsemberis S, Gulcur L, Nakae M. Housing First, Consumer Choice, and Harm Reduction for Homeless Individuals With a Dual Diagnosis. American Journal of Public Health. 2004;94(4):651-656. doi: 10.2105/ ajph.94.4.651.

51. Moreira RS, Mendes APT, Sciammarella APDO, Amado F. População em situação de rua: direitos humanos, políticas públicas e programas de housing first. Rio de Janeiro: Escola de Direito do Rio de Janeiro, Fundação Getúlio Vargas; 2017.

52. Castel R. As metamorfoses da questão social: uma crônica do salário. Petrópolis: Vozes; 1998.

53. Bastos FIPM, Bertoni N. Pesquisa Nacional sobre o uso de crack: quem são os usuários de crack e/ou similares do Brasil? quantos são nas capitais brasileiras? [Internet]. Rio de Janeiro: Instituto de Comunicação e Informação Científica e Tecnológica em Saúde/Fundação Oswaldo Cruz; 2014 [citado 17 jun 2019]. Disponible en: https://tinyurl.com/y7ugm2jx.

54. Bourgois P. Crack and the political economy of social suffering. Addiction Research and Theory. 2003;11(1):3137. doi: $10.1080 / 1606635021000021322$.

55. Escohotado A. Historia general de las drogas. Madrid: Espasa Calpe; 2005.

56. Garcia M. O uso problemático de crack e a classe média. En: Souza J, (ed.). Crack e exclusão social. Brasília: Ministério da Justiça e Cidadania, Secretaria Nacional de Políticas sobre Drogas; 2016. p. 103-118.

57. Varanda W, Adorno RCF. Descartáveis urbanos: discutindo a complexidade da população de rua e o desafio para políticas de saúde. Saúde e Sociedade. 2004;13(1):5669. doi: 10.1590/S0104-12902004000100007.

58. Zafiropoulos M, Pinell P. Drogas, desclassificação e estratégias de desqualificação. En: Pinell $\mathrm{P}$, (ed.). Análise sociológica das políticas de saúde. Rio de Janeiro: Editora Fiocruz; 2010. p. 143-176.

59. Goltzman PM. Memorias del Encuentro Intervenciones desde la Reducción de Daños: perspectivas y desafíos actuales [Internet]. Ciudad Autónoma de Buenos Aires: Intercambios Asociación Civil; 2016 [citado 17 jun 2019]. Disponible en: https://tinyurl. com/y8xe54r6.

60. Galante A. Experiencias de atención a usuarios de drogas en América Latina. En: Touzé G, Goltzman P (comps.). América Latina debate sobre drogas - I y II Conferencias Latinoamericanas sobre Políticas de Drogas. Buenos Aires: Intercambios Asociación Civil, Facultad de Ciencias Sociales, Universidad de Buenos Aires; 2011. p. 129-130.

61. Seffner F. Identidade de gênero, orientação sexual e vulnerabilidade social: pensando algumas situações brasileiras. En: Venturi G, Bokany V. Diversidade Sexual e homofobia no Brasil. São Paulo: Fundação Perseu Abramo; 2011. p. 39-50.

62. Moraes M. Gênero e usos de drogas: porque é importante articular esses temas? En: Moraes M, Castro R Petuco D, (orgs.). Gênero e drogas: contribuições para uma atenção integral à saúde. Recife: Instituto PAPAl; 2010. p. $15-20$.

63. Couto MT, Gomes R. Homens, saúde e políticas públicas: a equidade de gênero em questão. Ciência \& Saúde Coletiva. 2012;17:2569-2578. doi: 10.1590/ S1413-81232012001000002.

64. Granja E, Gomes R, Medrado B, Nogueira C. O (não) lugar do homem jovem nas políticas de saúde sobre drogas no Brasil: aproximações genealógicas. Ciência \& Saúde Coletiva. 2015;20(11):3447-3455. doi: 10.1590/1413-812320152011.19972014.

65. Mello L, Gonçalves E. Diferença e interseccionalidade: notas para pensar práticas em saúde. Revista Cronos. 2010;11(2):163-173.

66. Silveira ML. Políticas Públicas de Gênero: Impasses e Desafios para Fortalecer a Agenda Política na Perspectiva da Igualdade. En: Godinho T, (ed.). Políticas públicas e igualdade de gênero. São Paulo: Coordenadoria Especial da Mulher; 2004. p. 65-76.

67. Castro MG. Políticas Publicas por Identidades e de Ações Afirmativas: Acessando gênero e raça, na classe, focalizando juventudes [Internet]. Portal de e-governo, inclusão digital e sociedade do conhecimento; 2006 [citado 17 jun 2019]. Disponible en: https://tinyurl.com/ y96wrhhk.

68. Lugones M. Subjetividad esclava, colonialidad de género, marginalidad y opresiones múltiples. En: Pensando los feminismos en Bolivia. La Paz: Serie Foros; 2012. p. 129-140.

69. Curiel Pichardo RYO. Descolonizando el Feminismo: Una perspectiva desde América Latina y el Caribe [Internet]. En: Primer Coloquio Latinoamericano sobre Praxis y Pensamiento Feminista. Buenos Aires; 
2009 [citado 17 jun 2019]. Disponible en: https://tinyurl. com/y773qpvt.

70. Waiselfisz JJ, Instituto Sangari, Rede de Informação Tecnológica Latino-America, Ministério da Justiça. Mapa da violência: os jovens da América Latina. Brasilia: Rede de Informação Tecnológica Latino-Americana; 2008.

71. Count the Costs. The war on drugs: Undermining Human Rights [Internet]. 2012 [citado 17 jun 2019]. Disponible en: https://tinyurl.com/y8g3dhp5.

72. Castro-Gómez S. Ciências sociais, violência epistêmica e o problema da 'invenção do outro'. En: Lander E, (ed.). A colonialidade do saber: eurocentrismo e ciências sociais. Perspectivas latino-americanas. Buenos Aires: CLACSO; 2005. p. 169-186.

73. Borges J. Encarceramento em massa. São Paulo: Pólen; 2019.

74. Ribeiro M, Dunn J, Sesso R, Dias AC, Laranjeira R. Causes of death among crack cocaine users. Revista Brasileira de Psiquiatria. 2006;28(3):196-202. doi: 10.1590/ S1516-44462006000300010.
75. Espinosa Y. La política sexual radical autónoma, sus debates internos y su crítica a la ideología de la diversidad sexual. En: Pensando los feminismos en Bolivia. La Paz: Conexión Fondo de Emancipación; 2012.

76. Dutra V, Henriques V. O poder discricionário dos agentes institucionais que lidam com usuários de crack: invisibilidade de classe e estigma de gênero. En: Souza J, (ed.). Crack e exclusão social. Brasília: Ministério da Justiça e Cidadania, Secretaria Nacional de Políticas sobre Drogas; 2016.

77. Ronzani TM, Furtado EF, Higgins-Biddle J. Stigmatization of alcohol and other drug users by primary care providers in Southeast Brazil. Social Science \& Medicine. 2009;69(7):1080-1084. doi: 10.1016/j.socscimed.2009.07.026.

78. Moraes M, Montenegro M, Medrado B, Romaní O. Los retos de la reducción de daños como política pública de drogas en Brasil. Psicologia em Pesquisa. 2015;9(2):148158. doi: 10.5327/Z1982-1247201500020005.

79. Boneti LW. Políticas Públicas, Direitos Humanos e Cidadania. Juris. 2006;26:189-204. doi: 10.14295/juris. v26i0.629.

\section{FORMA DE CITAR}

Marques ALM, Couto MT. Políticas de drogas en el contexto brasileño: un análisis interseccional de "Cracolandia" en San Pablo, Brasil. Salud Colectiva. 2020;16:e2517. doi: 10.18294/sc.2020.2517.

Recibido: 31 ago 2019 | Versión final: 1 jun 2020 | Aprobado: 16 jun 2020 | Publicado en línea: 17 jul 2020

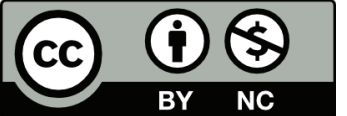

Esta obra está bajo una licencia de Creative Commons Reconocimiento-NoComercial 4.0

Internacional. Reconocimiento - Permite copiar, distribuir y comunicar públicamente la obra.

A cambio, se debe reconocer y citar al autor original. No Comercial - Esta obra no puede ser

utilizada con finalidades comerciales, a menos que se obtenga el permiso. 